\title{
A reconstituted HDL containing V156K or R173C apoA-I exhibited anti-inflammatory activity in apo-E deficient mice and showed resistance to myeloperoxidase-mediated oxidation
}

\author{
Kyung-Hyun $\mathrm{Cho}^{1,3}$ and Jae-Ryong $\mathrm{Kim}^{2}$ \\ 'School of Biotechnology \\ Aging-associated Vascular Disease Research Center \\ Yeungnam University \\ Gyeongsan 712-749, Korea \\ ${ }^{2}$ Department of Biochemistry and Molecular Biology \\ Aging-associated Vascular Disease Research Center \\ Yeungnam University \\ Daegu 705-717, Korea \\ ${ }^{3}$ Corresponding author: Tel, 82-53-810-3026; \\ Fax, 82-53-814-3026; E-mail, chok@yu.ac.kr \\ DOI 10.3858/emm.2009.41.6.047
}

Accepted 20 January 2009

Abbreviations: apo, apolipoprotein; GOT, glutamic oxaloacetic transaminase; GPT, glutamic pyruvic transaminase; LPO, lipid hydroperoxide; MPO, myeloperoxidase; rHDL, reconstituted high-density lipoprotein; TG, triglyceride

\begin{abstract}
It has been hypothesized that blood infusion of reconstituted $\mathrm{HDL}(\mathrm{rHDL})$ is a possible therapeutic strategy for the treatment of coronary artery disese. To compare short-term anti-inflammatory activity of wildtype (WT) apoA-I and point mutants, each rHDL containing WT, V156K, or R173C was infused into apo-E deficient atherosclerotic mice. Each rHDL was injected via the tail vein at a dosage of $120 \mathrm{mg} / \mathrm{kg}$ of body weight in $0.4 \mathrm{ml}$ of tris-buffered saline (TBS), and blood was then collected at 24 and $48 \mathrm{~h}$ post-injection. Although regression activity was observed in each of the rHDL infused groups, a $30 \%$ reduction in the lipid-stained area of the aortic sinus was observed in the V156K and R173C-rHDL groups when compared to that of the WT-rHDL group, and this reduction was well correlated with an approximately $60 \%$ reduction in the accumulation of macrophages in the lesion area. Additionally, the groups that received the V156K and R173C-rHDL treatments showed smaller increases in the GOT, GPT, interleukin-6, myeloperoxidase (MPO) and lipid hydroperoxide (LPO) serum levels than those that received the WT-rHDL treatment. In addition, the strongest serum paraoxonase and ferric reducing ability was ob-
\end{abstract}

served in the V156K and R173C-rHDL groups. In vitro nitration and chlorination of apoA-I by MPO treatment revealed that V156K-rHDL and R173C-rHDL were less susceptible to chlorination. Furthermore, rHDL treatment inhibited cellular uptake of oxidized LDL by macrophage cells and the production of proatherogenic species in culture media. In conclusion, blood infusions of the rHDLs exerted in vivo regression activity with anti-inflammatory and antioxidant activity in apo-E deficient mice and THP-1 cells, especially in those that were treated with V156K and R173C apoA-I.

Keywords: apolipoprotein A-I; apolipoproteins E; atherosclerosis; inflammation; lipoproteins, HDL

\section{Introduction}

It has been firmly established that serum HDL plays a protective role against the development of cardiovascular disease (Gordon et al., 1977; LinselNitschke and Tall, 2005) under clinical conditions, as the result of its anti-atherogenic and anti-inflammatory properties (Barter et al., 2004). As Ross defined atherosclerosis is an inflammatory disease (Ross, 1999), an atherosclerotic lesions were formed by interactions among endothelial cells, monocytes, and $T$ cells under high level of oxidized LDL (oxLDL). Apoplipoprotein (apo) A-I is a principal protein component of HDL that is known to perform a crucial role in the reverse cholesterol transport pathway through its anti-oxidant and anti-inflammatory activities (Barter et al., 2004; Ansell et al., 2005). Coronary heart disease ApoA- I-directed therapies, including the intravenous infusion of apoA-I/phospholipid, have been shown to have an anti-atherosclerotic effect that also stimulates cholesterol efflux and/or an atheroma regression effect in mice (Alam et al., 2001), rabbit (Miyazaki et al., 1995), and human models (Nanjee et al., 1999, 2001). Due to these findings, apoA-I and HDL are now widely believed to constitute an emerging therapeutic target for the treatment of coronary artery disease using a process referred to as HDL-therapy (Newton and Krause, 2002; Navab et al., 2004). The most prominent variant of apoA-I in the context of HDL-therapy is R173C-apoA-I (apoA-I Milano), which has been shown to have therapeutic potential in a series of in vivo 
Table 1. Experimental design of blood infusion into apo-E deficient mouse ${ }^{1}$.

\begin{tabular}{|c|c|c|c|c|c|c|c|}
\hline Group & Diet & $\begin{array}{l}\text { Injected rHDL } \\
\text { conc. (mg of } \\
\text { apoA-I) in TBS }\end{array}$ & $\mathrm{N}$ & $\begin{array}{l}\text { Body weight at } \\
\text { injection }^{2}(\mathrm{~g})\end{array}$ & $\begin{array}{l}\text { Total cholesterol } \\
\text { concentration before } \\
\text { injection ( } \mathrm{mg} / \mathrm{dl})\end{array}$ & $\begin{array}{c}\text { Injected rHDL amount }{ }^{2} \\
\text { (mg of apoA-l/kg of } \\
\text { body weight) }\end{array}$ & $\begin{array}{l}\text { Endotoxin in } \\
\text { rHDL (E.U./ml) }\end{array}$ \\
\hline WT & $W D^{3}$ & $\begin{array}{l}\text { Wildtype } \\
(10 \mathrm{mg} / \mathrm{ml})\end{array}$ & 10 & $27.6 \pm 1.9$ & $1,254 \pm 70$ & $\begin{array}{c}120 \mathrm{mg} / \mathrm{kg} \text { of } \\
\text { body weight }\end{array}$ & $3.1 \pm 0.3$ \\
\hline V156K & WD & $\begin{array}{l}\text { V156K } \\
(10 \mathrm{mg} / \mathrm{ml})\end{array}$ & 10 & $26.9 \pm 1.6$ & $1,183 \pm 146$ & $\begin{array}{l}120 \mathrm{mg} / \mathrm{kg} \text { of } \\
\text { body weight }\end{array}$ & $3.2 \pm 0.3$ \\
\hline $\mathrm{R} 173 \mathrm{C}$ & WD & $\begin{array}{l}\mathrm{R} 173 \mathrm{C} \\
(8.8 \mathrm{mg} / \mathrm{ml})\end{array}$ & 10 & $27.0 \pm 3.8$ & $1,227 \pm 104$ & $\begin{array}{l}120 \mathrm{mg} / \mathrm{kg} \text { of } \\
\text { body weight }\end{array}$ & $3.3 \pm 0.2$ \\
\hline TBS & WD & buffer $0.4 \mathrm{ml}$ & 6 & $26.2 \pm 2.9$ & $1,200 \pm 40$ & $0.4 \mathrm{ml}$ of TBS & $0.1 \pm 0.1$ \\
\hline
\end{tabular}

${ }^{1}$ Male apo-E deficient mice (Jackson Lab,Tm1Unc, JAX \# 1409109 ) were fed a western diet for 15 weeks. ${ }^{2} \mathrm{~A}$ single injection (in $0.4 \mathrm{ml}$ of TBS) was administered at $0 \mathrm{~h}$ and blood was collected at 24 and $48 \mathrm{~h}$ post injection. ${ }^{3} \mathrm{WD}$, western diet; $0.15 \%$ cholesterol and $15 \%$ lard in CRF-1 (Oriental Yeast, Japan). TBS, tris buffered saline.

experiments in both animals (Shah et al., 1998, 2001; Chiesa et al., 2002; Kaul et al., 2003) and humans (Nissen et al., 2003). Among these reports, the clinical data reported by the Nissen group suggested that a blood infusion of the phospholipidapoA-I Milano $_{\text {complex resulted in significant regression }}$ activity of up to $4 \%$ above baseline levels, as measured by intravascular ultrasound.

We previously reported several point mutants of apoA-I in helix 6 (143-164 amino acid) domain. Among the mutants, the $\mathrm{V} 156 \mathrm{~K}$ protein of apoA-I showed unique structural and functional properties in vitro (Han et al., 2005), and V156K-rHDL has been shown to exert anti-oxidant effects in hypercholesterolemic C57BL/6 mice (Cho et al., 2006, 2007). In this study, the anti-atherosclerotic effects of V156K-rHDL were assessed in apo-E deficient mice to verify the regression effect and the anti-inflammatory and anti-oxidant activity in vivo under hypothesis of HDL/apoA-I infusion therapy. In addition, in vitro experiments were conducted to evaluate myeloperoxidase (MPO) mediated oxidation and cellular uptake of oxidized LDL (oxLDL) in macrophages to better understand the mechanism by which the therapeutic effect of apoA-I and its mutants occurs. Because MPO is a source of oxidative stress in human artery walls and is recognized as a potential marker of cardiovascular disease (Nicholls and Hazen, 2005), the degree of oxidation of apoA-I in the lipid-bound state that was mediated by MPO was compared between WT, $\mathrm{V} 156 \mathrm{~K}$, and R173C in vitro. Additionally, because phagocytosis of oxLDL by macrophages is the initial step in foam cell formation and atherosclerosis, the cellular uptake of oxLDL by macrophages in the presence of rHDL was evaluated.

\section{Results}

\section{Infusion of rHDL improved the ratio of HDL-cholesterol}

After 15 weeks on a Western Diet, all of the mice had high serum total cholesterol (TC) levels that

Table 2. Serum profile of apo-E deficient mice after blood infusion of $\mathrm{rHDL}^{1,2}$.

\begin{tabular}{ccccccccccc}
\hline & $\begin{array}{c}\text { Group } \\
(\mathrm{n})\end{array}$ & $\mathrm{n}$ & $\begin{array}{c}\mathrm{TC} \\
(\mathrm{mg} / \mathrm{dl})\end{array}$ & $\begin{array}{c}\mathrm{HDL}-\mathrm{C} \\
(\mathrm{mg} / \mathrm{dl})\end{array}$ & $\begin{array}{c}\mathrm{HDL}-\mathrm{Cl} \\
\mathrm{TC}(\%)\end{array}$ & $\begin{array}{c}\text { TG } \\
(\mathrm{mg} / \mathrm{dl})\end{array}$ & $\begin{array}{c}\text { GOT } \\
(\mathrm{U} / \mathrm{L})\end{array}$ & $\begin{array}{c}\text { GPT } \\
(\mathrm{U} / \mathrm{L})\end{array}$ & $\begin{array}{c}\text { IL-6 } \\
(\mathrm{pg} / \mathrm{ml})\end{array}$ & $\begin{array}{c}\text { Uric acid } \\
(\mathrm{mg} / \mathrm{dl})\end{array}$ \\
\hline $24 \mathrm{~h}$ & WT & 10 & $1,333 \pm 96^{\mathrm{a}}$ & $20 \pm 3^{\mathrm{a}}$ & $1.5^{\mathrm{a}}$ & $162 \pm 36^{\mathrm{a}}$ & $744 \pm 226^{\mathrm{a}}$ & $122 \pm 58^{\mathrm{a}}$ & $130 \pm 26^{\mathrm{a}}$ & - \\
post & V156K & 10 & $1,206 \pm 66^{\mathrm{a}}$ & $19 \pm 2^{\mathrm{a}}$ & $1.6^{\mathrm{a}}$ & $133 \pm 47^{\mathrm{a}}$ & $321 \pm 53^{\mathrm{b}}$ & $26 \pm 13^{\mathrm{b}}$ & $139 \pm 36^{\mathrm{a}}$ & - \\
injection & $\mathrm{R} 173 \mathrm{C}$ & 10 & $1,285 \pm 95^{\mathrm{a}}$ & $24 \pm 5^{\mathrm{a}}$ & $1.9^{\mathrm{a}}$ & $162 \pm 55^{\mathrm{a}}$ & $420 \pm 237^{\mathrm{ab}}$ & $42 \pm 46^{\mathrm{ab}}$ & $139 \pm 44^{\mathrm{a}}$ & - \\
& TBS & 6 & $1,530 \pm 78^{\mathrm{b}}$ & $10 \pm 3^{\mathrm{b}}$ & $0.7^{\mathrm{b}}$ & $125 \pm 15^{\mathrm{b}}$ & $235 \pm 32^{\mathrm{c}}$ & $25 \pm 11^{\mathrm{b}}$ & $68 \pm 22^{\mathrm{b}}$ & - \\
\hline 48 h & WT & 10 & $1,285 \pm 112^{\mathrm{a}}$ & $20 \pm 7^{\mathrm{a}}$ & $1.6^{\mathrm{a}}$ & $163 \pm 8^{\mathrm{a}}$ & $438 \pm 80^{\mathrm{a}}$ & $34 \pm 16^{\mathrm{a}}$ & $104 \pm 76^{\mathrm{a}}$ & $3.8 \pm 0.03^{\mathrm{a}}$ \\
post & V156K & 10 & $1,183 \pm 47^{\mathrm{a}}$ & $22 \pm 5^{\mathrm{a}}$ & $1.9^{\mathrm{a}}$ & $92 \pm 10^{\mathrm{b}}$ & $380 \pm 62^{\mathrm{a}}$ & $23 \pm 2^{\mathrm{b}}$ & $34 \pm 29^{\mathrm{b}}$ & $2.2 \pm 0.03^{\mathrm{b}}$ \\
injection & $\mathrm{R} 173 \mathrm{C}$ & 10 & $1,240 \pm 173^{\mathrm{a}}$ & $23 \pm 4^{\mathrm{a}}$ & $1.9^{\mathrm{a}}$ & $97 \pm 27^{\mathrm{b}}$ & $385 \pm 116^{\mathrm{a}}$ & $23 \pm 7^{\mathrm{ab}}$ & $64 \pm 50^{\mathrm{ab}}$ & $1.8 \pm 0.03^{\mathrm{b}}$ \\
& TBS & 6 & $1,352 \pm 187^{\mathrm{a}}$ & $12 \pm 3^{\mathrm{b}}$ & $0.9^{\mathrm{b}}$ & $102 \pm 10^{\mathrm{b}}$ & $365 \pm 205^{\mathrm{a}}$ & $47 \pm 17^{\mathrm{b}}$ & $31 \pm 21^{\mathrm{b}}$ & $4.2 \pm 0.04^{\mathrm{a}}$ \\
\hline
\end{tabular}

${ }^{1}$ Data were expressed as the mean \pm S.D. ${ }^{2}$ Means in the same column not sharing a common superscript are significantly different $(P<0.05)$ between groups. GOT, glutamic oxaloacetic transaminase; GPT, gamma-glutamic pyruvic transaminase; HDL, high density lipoprotein; IL-6, interleukin-6; TBS, tris-buffered saline; TC, total cholesterol; TG, triacylglyceride. 
were in the range of $1,100-1,300 \mathrm{mg} / \mathrm{dl}$ of TC (Table 1) and $10 \pm 3 \mathrm{mg} / \mathrm{dl}$ of HDL-C, with a $0.7-0.8 \% \mathrm{HDL}-\mathrm{C} / \mathrm{TC}$ ratio. At 24 and $48 \mathrm{~h}$ after $\mathrm{rHDL}$ infusion, the concentration of total serum cholesterol did not change significantly in a similar range as was observed prior to injection (Table 2). Moreover, the ratio of HDL-C/TC in the rHDL injected group was $1.6-1.9 \%$, at $48 \mathrm{~h}$ post-injection, which was elevated when compared to that of the TBS-injected group, which had an HDL-C/TC elevation of $0.9 \%$. Taken together, these results suggest that HDL-C accumulated rapidly as a result of the rHDL treatment. Although we did not measure the serum cholesterol levels at $1 \mathrm{~h}$ after injection, the values at 24 and $48 \mathrm{~h}$ did not fluctuate (Table 2). However, the TG concentration was elevated to approximately 133-162 $\mathrm{mg} / \mathrm{dl}$ at $24 \mathrm{~h}$, and then decreased to $92-97 \mathrm{mg} / \mathrm{dl}$ at $48 \mathrm{~h}$ for V156K-rHDL and R173C-rHDL group. Throughout the $48 \mathrm{~h}$ period, no significant changes were observed in the TC and HDL-C levels in any of the groups.

\section{Decreased inflammatory parameters in V156K or R173C-rHDL infused group}

Among the rHDL infused groups, the V156K-rHDL group showed the smallest serum glutamic oxaloacetic transaminase (GOT) and gamma-glutamic pyruvic transaminase (GPT) values, approximately $321 \pm 53 \mathrm{U} / \mathrm{L}$ and $26 \pm 13 \mathrm{U} / \mathrm{L}$, respectively, at 24 h post-injection (Table 2). However, the WT-rHDL injected group showed the highest serum GOT and
GPT values, $744 \pm 226$ and $122 \pm 58 \mathrm{U} / \mathrm{L}$, respectively, and the TBS-injected group showed values of $235 \pm 32$ and $25 \pm 11 \mathrm{U} / \mathrm{L}$, respectively. In addition, the values of the group injected with R173C-rHDL were slightly higher than those of the V156K-rHDL group. Although the entire rHDL-injected group showed a similar range of serum IL-6 levels at $24 \mathrm{~h}$ (approximately 130-139 $\mathrm{pg} / \mathrm{ml}$ ), the TBS-injected group displayed levels of $68 \pm 22 \mathrm{pg} / \mathrm{ml}$. At $48 \mathrm{~h}$ post-injection, however, the V156K-rHDL injected group showed minimal values of $34 \pm 29 \mathrm{pg} / \mathrm{ml}$, whereas the WT-rHDL and R173C-rHDL injected groups were found to have levels of $104 \pm 76$ and $64 \pm 50 \mathrm{pg} / \mathrm{ml}$, respectively. These results indicate that V156K-rHDL and R173C-rHDL infusion might have a greater effect than WT-rHDL infusion with regard to the attenuation of a putative process of inflammatory cytokine production and the attendant cascade.

As shown in Table 2, the serum uric acid level was increased by up to $4.2 \pm 0.5 \mathrm{mg} / \mathrm{dl}$ as a result of $\mathrm{HCHF}$ consumption (TBS group), which is much greater than the concentration of $1.5 \pm 0.3 \mathrm{mg} / \mathrm{dl}$ that was observed in the normal C57BL/6J mice sera. However, the uric acid levels in the sera obtained from the V156K-rHDL and R173C-rHDL infused groups were $2.2 \pm 0.2$ and $1.8 \pm 0.2 \mathrm{mg} / \mathrm{dl}$, respectively, whereas that of the sera obtained from the WT-rHDL infused group was $3.8 \pm 0.5$ $\mathrm{mg} / \mathrm{dl}$ at $48 \mathrm{~h}$ post infusion. Recently, an increased serum uric acid level has been recognized as an
A

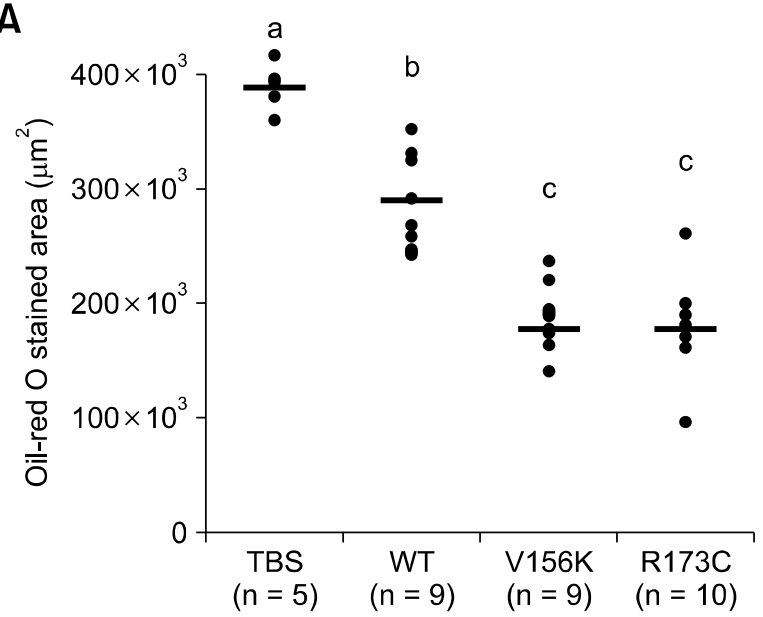

B

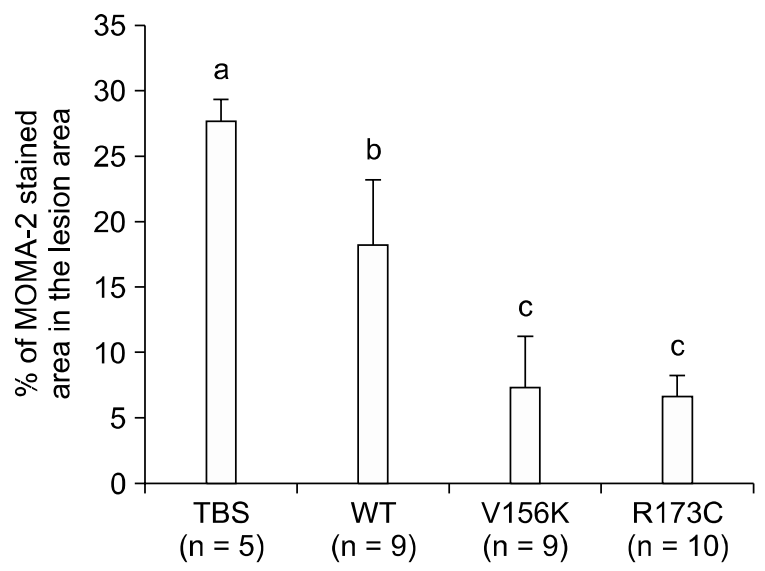

Figure 1. Anti-atherosclerotic effect of rHDL infusion. (A) Reduction of fatty-streak lesion area. The mean size of the lipid stained area was calculated and quantified via computer-assisted morphometry (Image Proplus). The mean values that do not share a common letter are significantly different between groups $(P<0.05)$. (B) Reduction of macrophage accumulation in the fatty streak lesions. Immunohistochemical lesion analysis for the visualization of macrophages was conducted using an anti-mouse macrophage antibody (MOMA-2, MCA519, Serotec). A fluorescence-labeled secondary antibody (Cy2-conjugated-anti-lgG) was used to visualize the detected macrophages in the lesion area under confocal microscopy (Zeiss, LSM510 meta). The proportional ratio of the MOMA-2 stained area to the Oil red $O$ stained area was then compared. The mean values that do not share a common letter are significantly different between groups $(P<0.05)$. 
inflammatory marker, which is strongly associated with coronary atherosclerosis and metabolic syndrome (Coutinho et al., 2007). Taken together, the changes of GOT, GPT, IL-6, and uric acid in serum were well correlated in regard to anti-inflammatory effect of the rHDL.

\section{Reduced fatty streak lesions and macrophage accumulation in rHDL infused group}

The consumption of a Western diet for 15 weeks resulted in the accretion of a thick, strong fatty streak lesion, as visualized by oil red-O/hematoxylin staining (Supplemental Data Figure S1A). When the thickness at the same position was compared, the TBS-injected group also had a well-developed lesion area, with the strongest intensity being observed in the lipid-stained area $\left(387,940 \pm 23,540 \mu^{2}\right)$, whereas the WT-rHDL group showed a decreased area $(268,420 \pm$ $41,000 \mu^{2}$ ). The V156K-rHDL and R173C-rHDL groups showed significantly reduced lipid areas of $188,890 \pm 43,000 \mu \mathrm{m}^{2}$ and $179,480 \pm 42,220 \mu \mathrm{m}^{2}$ (Figure 1A), respectively. Consistent with the results obtained from the oil red-O/hematoxylin staining, the MOMA-2 stained region in the fatty streak lesion was also reduced in size in the WT-rHDL group, V156K-rHDL, and R173C-rHDL groups, at $18.2 \pm 5 \%, 7.3 \pm 4 \%$, and $6.6 \pm 2 \%$, correspondingly, as compared with that the TBS group (27 \pm $2 \%$ ), as shown in Figure $1 \mathrm{~B}$ and Supplemental Data Figure S1B. Taken together, these results show that WT-rHDL had regression activity, which is shown by a $30 \%$ reduction in lipid area as com- pared to the buffer, however, this effect was not more substantial than was observed in the V156K-rHDL and R173C- rHDL groups.

\section{Immunodectetion of apoA-I in mice sera}

At $48 \mathrm{~h}$ post-injection, ELISA analysis of samples obtained from mice treated with human proapoA-I (29 kDa) detected WT-rHDL at very low levels, (< $1 \mathrm{mg} / \mathrm{dl}$ ), whereas V156K-rHDL and R173C-rHDL were detected at levels of approximately 14-16 $\mathrm{mg} / \mathrm{dl}$ (Figure 2A). No human proapoA-I was detected in the TBS-injected group, as the ELISA kit was unable to detect mouse apoA-I, but was able to detect only human apoA-I. This indicates that WT-rHDL was disappeared more quickly than V156K-rHDL and R173C-rHDL in mice sera. This result was verified via Western blot analysis (Figure 2B) using the anti-apoA-I antibody, which revealed that the human WT-proapoA-I band (29 $\mathrm{kDa}$ ) was detected distinctly at $24 \mathrm{~h}$ after treatment, but that this band disappeared almost completely at $48 \mathrm{~h}$.

\section{Serum PON activity was enhanced in V156K and R173C-rHDL group}

As shown in Figure 3A, all rHDL groups showed a higher serum PON activity than that of TBS group, and the rHDL groups showed a stronger PON activity at $24 \mathrm{~h}$ than at $48 \mathrm{~h}$. Sera from mice that were infused with V156K-rHDL showed the strongest activity (98 \pm 5 and $72 \pm 4 \mathrm{U} / \mathrm{L}$ at 24 and 48 $\mathrm{h}$ post-injection, respectively). R173C-rHDL and
A

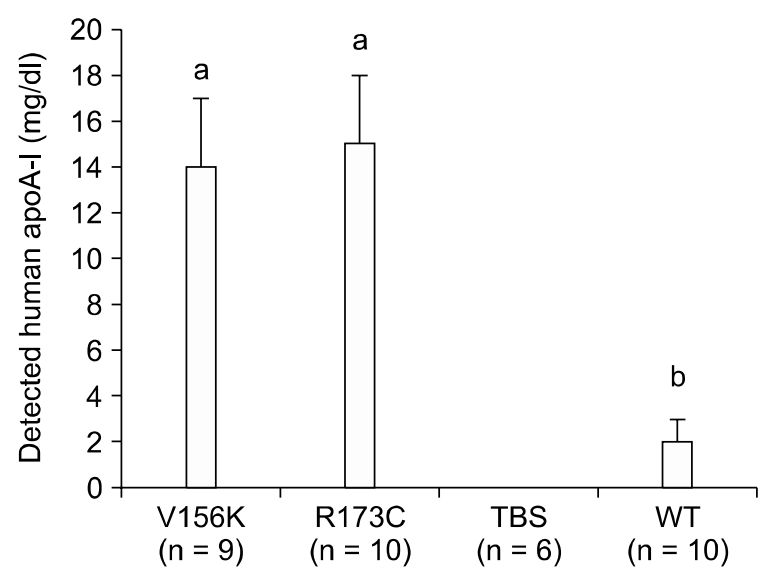

B

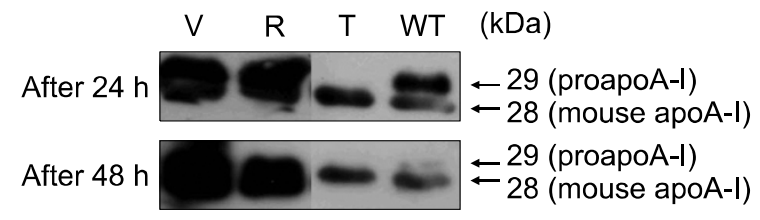

Figure 2. Immunodetection of human apoA-I and mouse apoA-I in the mice sera. Immunodetection indicated that V156K and R173C bands were detected much more that of WT-apoA-I band at $48 \mathrm{~h}$ post-infusion, whereas no significant change was found in mouse apoA-I level among all groups. The V156K and R173C levels were determined to be similar (around $15 \mathrm{mg} / \mathrm{dl}$ ) $48 \mathrm{~h}$ after infusion using ELISA-based quantification as described in the text (A). Human proapoA-I and mouse apoA-I were detected as bands of 29 and $28 \mathrm{kDa}$, respectively (B). The mean values not sharing a common letter are significantly different between groups $(P<0.01)$. 
A

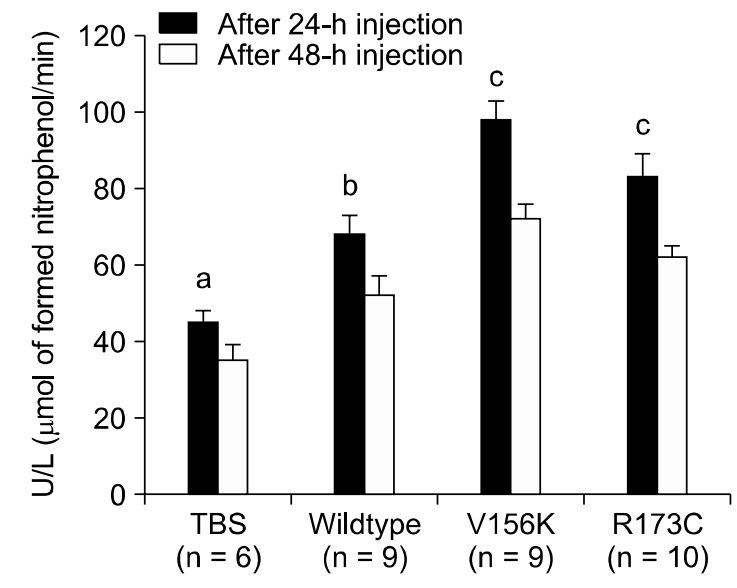

PON-1

$(43 \mathrm{kDa})$

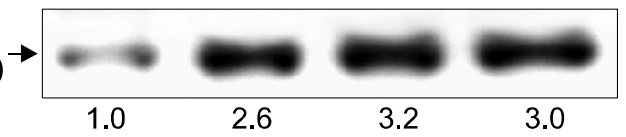

B
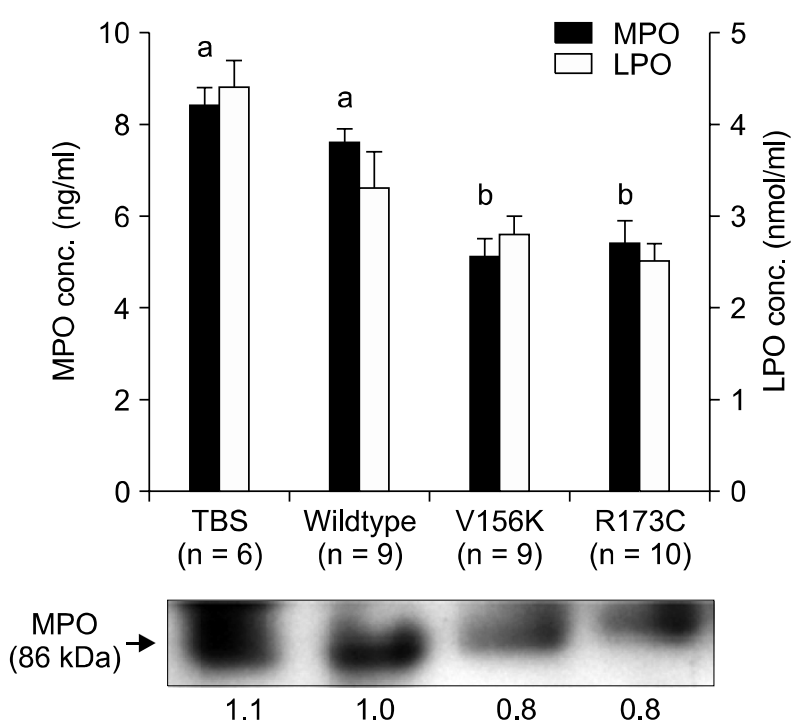

Figure 3. Paraoxonase activity and inflammatory markers in mice sera. (A) Paraoxonase activity. Equally diluted serum (20 $\mu \mathrm{l}, 20 \mathrm{mg} / \mathrm{ml})$ was added to $230 \mu \mathrm{l}$ of the paraoxon-ethyl (Sigma Cat\# D-9286) containing solution (90 mM Tris-HCl/3.6 mM NaCl/2 mM CaCl $2, \mathrm{pH} 8.5$ ). A PON-1 activity of $1 \mathrm{U} / \mathrm{L}$ is defined as $1 \mathrm{mmol}$ of $p$-nitrophenol formed per minute. The mean values not sharing a common letter are significantly different between groups $(P<$ 0.01). The bottom photo shows the immunodetected band of PON from pooled sera of each group. Lower numbers indicate the relative intensity of the detected band, as compared with the TBS-injected sera. (B) Serum concentration of myeloperoxidase (MPO) and lipid hydroperoxide (LPO) were reduced in the rHDL infused groups. Serum MPO and LPO were determined using an commercially available assay kit as described in text. The bottom photo shows the immunodetected band of MPO from pooled sera of each group, which was equally diluted ( $5 \mathrm{mg}$ of protein/ml). The lower numbers indicate the relative band intensity $(\mathrm{BI})$, as compared with that of TBS-injected sera. The mean values not sharing a common letter are significantly different between groups $(P<0.05)$.

WT-rHDL groups showed weaker activity than that of V156K-rHDL group, $83 \pm 6$ and $68 \pm 5 \mathrm{U} / \mathrm{L}$, respectively, while TBS group showed only $45 \pm 3$ $U / L$. The enzyme activity was well correlated with the immunodetection of PON-1 band $(43 \mathrm{kDa})$, as shown in bottom of Figure $3 \mathrm{~A}$. At $48 \mathrm{~h}$ postinjection, all of the rHDL groups showed approximately 3-fold higher band intensity than the TBS-injected group. Although the difference was not remarkable, the V156K-rHDL and R173C-rHDL group showed the highest detection level (band intensity, $\mathrm{BI}=3.2$ and 3.0 , respectively) among the $\mathrm{rHDL}$ groups.

\section{Serum MPO and LPO level was decreased by rHDL infusion}

ELISA-based detection revealed that V156K-rHDL or R173C-rHDL group showed a lower level of serum MPO than that of TBS or WT-rHDL group at $48 \mathrm{hr}$ post-injection. Specifically, the serum MPO level in apo-E deficient mice was increased around 7 -fold; TBS group showed up to $8.4 \pm 0.4 \mathrm{ng} / \mathrm{ml}$, whereas the HCHF consumed normal C57BL/6 mouse serum showed only $1.2 \pm 0.2 \mathrm{ng} / \mathrm{ml}$. The V156K-rHDL and R173C-rHDL group exhibited lower levels of $5.1 \pm 0.4$ and $5.4 \pm 0.3 \mathrm{ng} / \mathrm{ml}$, respectively, than level of WT-rHDL with $7.6 \pm 0.3$ $\mathrm{ng} / \mathrm{ml}$. This result suggests that the $\mathrm{rHDL}$ containing V156K or R173C could relieve the increase of serum MPO level in response to oxidative stress induced by the long-term HCHF consumption (Figure 3B). The immunodetection results corroborated the results of the ELISA, which showed that V156K-rHDL or R173C-rHDL infused sera had $20 \%$ lower MPO band intensity $(B I=0.8)$ than that of WT-rHDL group $(\mathrm{BI}=1.0)$ as shown in bottom of Figure 3B.

The serum LPO level was significantly lower in the rHDL infused groups, with the WT-rHDL group showing a LPO level that was $25 \%$ lower than that of the TBS group and the V156K-rHDL and R173C-rHDL infused groups showing LPO levels that were $36-44 \%$ lower than that of the TBS group (Figure 3B). Taken together, these results suggest that the regression effect induced by rHDL may be linked to the decreased serum MPO and LPO levels and enhanced the antioxidant ability of the sera.

\section{V156K-rHDL infused sera showed the strongest antioxidant ability}

The most profound antioxidant activity observed was the serum ferric removal ability of the V156K group, 
as shown in Figure 4. The absorbance values at 593 $\mathrm{nm}\left(\mathrm{A}_{593}\right)$ of sera collected at $24 \mathrm{~h}$ post-injection from V156K group were found to be $42 \%$ greater than the initial absorbance values, whereas the absorbance values of sera collected from the WT and R173C groups increased by only $20 \%$ and $30 \%$, respectively (panel A). Sera of the V156K group at $48 \mathrm{~h}$ post-injection continued to show the highest activity, with an increase of up to $40 \%$ being observed in the $A_{593}$ value, whereas the $A 593$ values of sera of WT-rHDL and R173C-rHDL group increased by 29 and $31 \%$, respectively (panel B). Additionally, the increase in the $A_{593}$ value of sera collected from the TBS group at 24 and $48 \mathrm{~h}$ post-injection was minimal, being only $17 \%$. These results are generally consistent with the results of our previous report, which found that V156K-rHDL infused sera exhibited the most potent antioxidant activity against copper-mediated oxidation, and that this antioxidant activity was derived from injected apoA-I protein, not from phospholipids (Cho et al., 2006, 2007). The highest increases in the level PON activity in V156K or R173C-rHDL groups also correlate well with the enhanced in vivo antioxidant activity observed in the FRAS assay, indicating that the better antioxidant activity of the rHDL group might be due, at least in part, to the stronger PON activity.

\section{V156K or R173C-rHDL was less susceptible to MPO-mediated oxidation in vitro}

MPO-mediated nitration revealed that WT-rHDL is slightly more sensitive than V156K-rHDL, as evidenced by production of 9.0 and $7.8 \mathrm{mM}^{\mathrm{ONOO}}$, respectively, after $4 \mathrm{~h}$ of incubation. Furthermore,
WT-rHDL showed more susceptibility to chlorination with production of $1.4 \mathrm{mM}$ of $\mathrm{HOCl}$ while V156K or R173C-rHDL produced $1.1 \mathrm{mM}$ of $\mathrm{HOCl}$. These differences in spectrophotometric data were further characterized by electrophoresis. As shown in Figure 5A, SDS-PAGE revealed that chlorinated WT-rHDL fragments were more degraded (around 22-23 kDa from comparison with molecular weight marker protein, indicated by the arrow head) than chlorinated V156K- or R173C-rHDL fragments. These results indicate that chlorination might be occurred at Tyr192, as reported by Shao et al. (2006) because Tyr192 is a selective target for MPO modification due to the presence of the YxxK motif.

Native electrophoresis of the chlorinated rHDL revealed that WT-rHDL showed particle sizes that had decreased from 98-100 $\AA$ to approximately 94-96 Å, as shown in Figure 5B. However, V156KrHDL and R173C-rHDL did not undergo the decrease of particle size after chlorination. These results showed that WT-rHDL is more susceptible to MPO-mediated oxidation, and that oxidation might influence its particle size and/or native mobility, whereas V156K-rHDL and R173C-rHDL were more resistant to the oxidation. This difference in susceptibility may be related to the different physiological activity that occurs in the blood of mice, such as different GOT, GPT, IL-6, and MPO levels.

\section{Cellular uptake of oxLDL was decreased by rHDL treatment}

After $48 \mathrm{~h}$ of incubation, the THP-1 cells were stained with oil red $O$ to evaluate the degree of
A

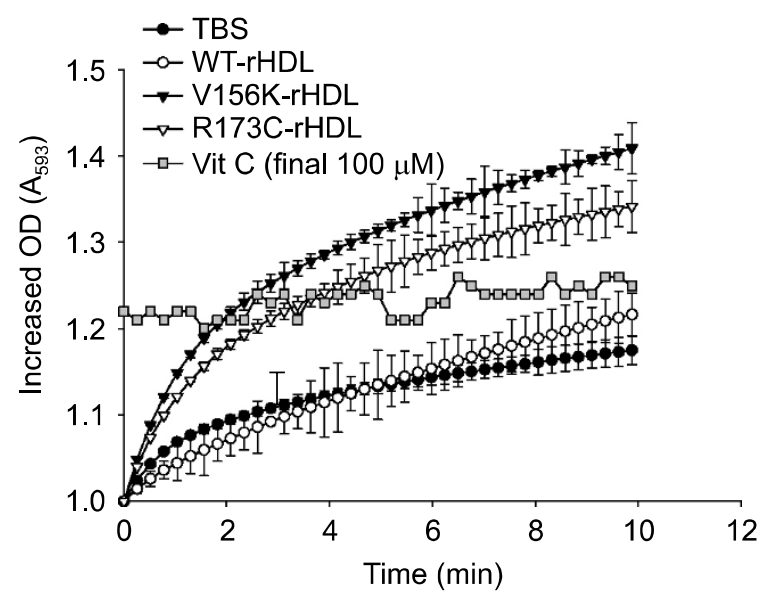

B

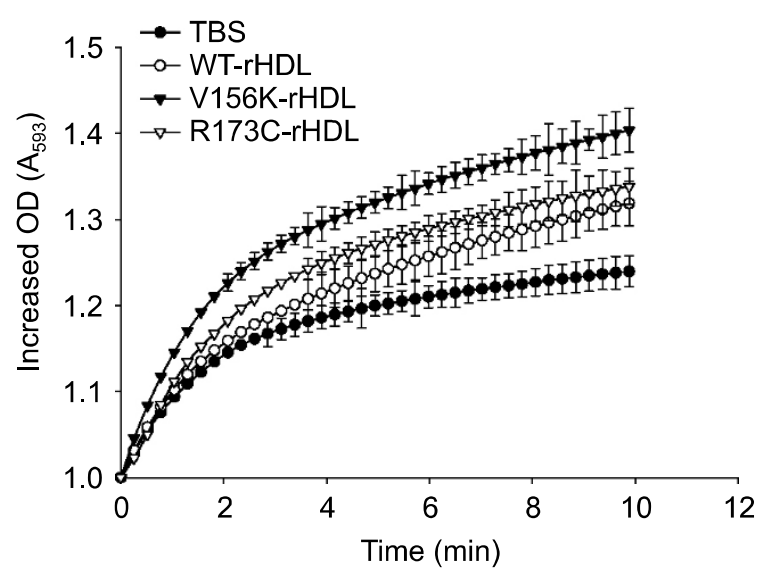

Figure 4. Rate of increase in absorbance at $593 \mathrm{~nm}$ of mice sera containing rHDL. V156K-rHDL infusion resulted in the most potent FRAS ability at 24 and $48 \mathrm{~h}$, whereas serum that was infused with WT-rHDL showed the lowest levels of FRAS ability. Vitamin C (final $100 \mu \mathrm{M}$ in PBS) was used as a positive control. Error bars indicate the SD of measurements that were taken in triplicate. 
lipid or LDL uptake into cells that occurred in the presence of each rHDL. As shown in Figure 6A, the strongest color intensity was observed in PBS treated cells, while the intensity was decreased in WT-rHDL treated cells. In addition, cells that were treated with V156K-rHDL or R173C-rHDL showed much less lipid stained color intensity than the WT-rHDL treated cells, indicating that rHDL treatment can inhibit the uptake of oxLDL. This result correlated well with the results of a TBARS assay of the culture media $(250 \mu \mathrm{l})$. As shown in Figure 6B, all of the media used to culture rHDL treated cells contained less MDA than media used to culture PBS treated cells. Furthermore, media that was used to culture V156K-rHDL and R173C-rHDL

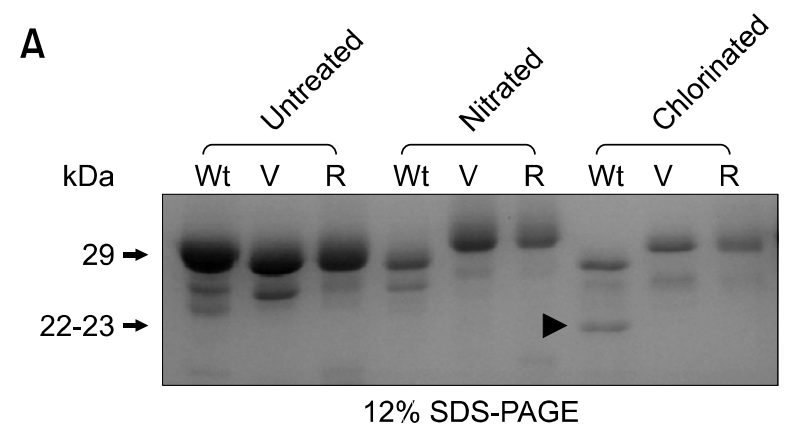

B

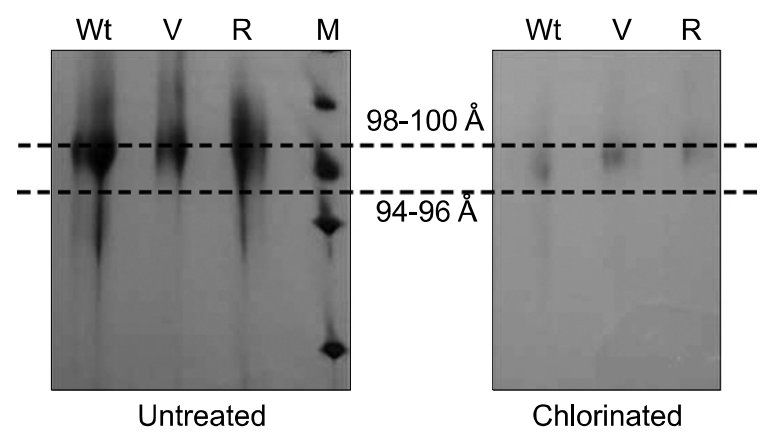

8-25\% Native PAGGE

Figure 5. Electrophoretic patterns of apoA-l in the lipid-bound state as a result of MPO-mediated oxidation in vitro. Lane Wt, WT-rHDL; V, V156K-rHDL; R, R173C-rHDL. (A) Electrophoretic patterns of apoA-I in rHDL with or without MPO treatment (12\% SDS-PAGE). The fragmented band as indicated by arrowhead approximately 22-23 kDa was found in chlorinated WT-rHDL, indicating that WT-apoA-I is more susceptible to the MPO-mediated oxidation than the mutants. Molecular weight of band was determined from comparison with standard proteins (BioRad, low-range molecular weight markers). (B) Change in particle size after chlorination (8-25\% native-PAGGE). The WT-rHDL particle size was reduced from 98-100 $\AA$ to $94-96 \AA$, whereas the particle sizes of V156K-rHDL and R173C-rHDL were unchanged. M, high molecular weight markers (AmershamPharmacia) indicating 122, 98, 81, and $71 \AA$ from top. treated cells showed $25 \%$ and $35 \%$ less MDA than media that was used to culture WT-rHDL treated cells. LPO level of $0.1 \mathrm{ml}$ of media that was used to culture rHDL treated cells was lower than that of media used to culture PBS treated cells. In addition, treatment with V156K-rHDL or R173C-rHDL resulted in a $20 \%$ decrease of LPO production when compared to treatment with WT-rHDL. These results suggest that rHDL treatment relieved production of MDA and LPO in the cell culture media, indicating that the antioxidant activity of rHDL was effective in macrophages grown in the presence of oxLDL. Taken together, these findings indicate that the ability of V156K or R173C-rHDL to inhibit further oxidation of LDL in culture media and cellular uptake of oxLDL by macrophages might be superior to that of WT-rHDL.

\section{Discussion}

The findings of this study suggest that rHDL exerts a regression effect in apo-E deficient mice with anti-oxidant and anti-inflammatory activity, especially V156K or R173C-rHDL. A number of studies regarding R173C-rHDL (ETC-216) have shown that a phospholipid complex with an apoA-I variant may exert a direct anti-atherosclerotic effect in a variety of animal models. However, these studies have primarily focused on R173C-rHDL, and their primary limitation was that they did not employ wildtype human apoA-I as a control for direct comparison (Shah et al., 1998, 2001; Chiesa et al., 2002; Kaul et al., 2003; Nissen et al., 2003). Our findings revealed that WT-rHDL also showed a regression effect; with the oil-red O-stained areas and the MOMA-2 stained areas being reduced in size by $31 \%$ and $34 \%$, respectively, when compared to the TBS group (Figure 1). Although this effect was smaller than that of the V156K or R173C-rHDL groups, these results clearly indicate that WT-rHDL produces an anti-atherosclerotic effect. This result is at least partially in agreement with those of a previous report that found that WT-rHDL infusion inhibits the early pro-oxidant and pro-inflammatory alterations induced by a periarterial collar in normocholesterolemic rabbits (Nicholls et al., 2005). Similarly, short term infusion of CSL-111, a synthetic rHDL manufactured from human plasma apoA-I and soybean phosphatidylcholine, exhibited significant improvement in plaque characteristics when compared to the results of treatment with a placebo in an ERASE (Effect of rHDL on atherosclerosissafety and efficacy) trial (Tardif et al., 2007).

The results of several in vitro experiments may explain the superior anti-atherosclerotic ability 
A
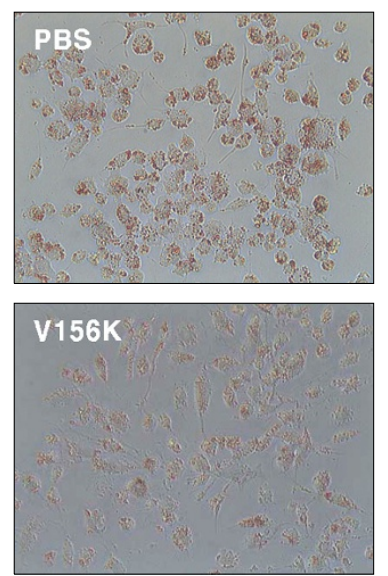

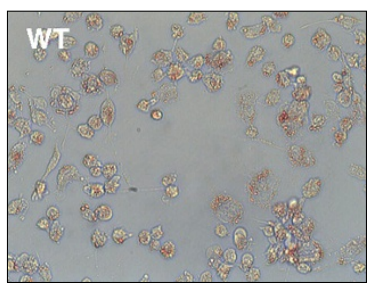

R173C
B

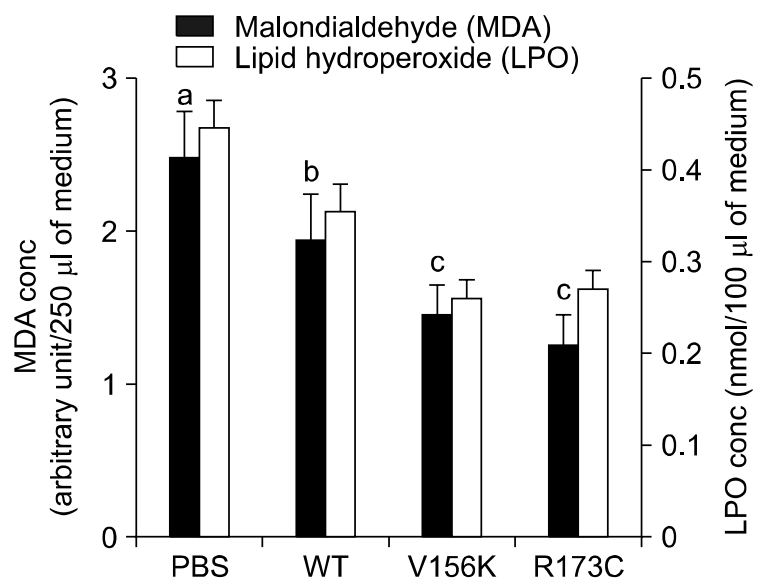

Figure 6. (A) Oil red $O$ staining of macrophages. PMA differentiated macrophages were incubated with $50 \mu \mathrm{l}$ of oxLDL (1 mg/ml), $50 \mu \mathrm{l}$ of $\mathrm{rHDL}(1.4$ $\mathrm{mg} / \mathrm{ml}$ ), and $400 \mu \mathrm{l}$ of RPMl1640 media. The extent of cellular uptake of lipids or LDL in macrophages was then compared by oil red $O$ staining as described in the text. The cells were then photographed using a Nikon Eclipse TE2000 microscope (Tokyo, Japan) at $600 \times$ magnification. (B) The cell media was analyzed by TBARS assay and the LPO assay kit (Calbiochem) to determine production of oxidized species. Malondialdehyde (MDA) was used as a standard and the extent of oxidation was expressed as MDA concentration (arbitrary unit, AU). The LPO level in $0.1 \mathrm{ml}$ of culture media was decreased in media that contained rHDL treated cells when compared to media that contained PBS treated cells. In addition, media that was used to culture cells treated with V156K-rHDL or R173C-rHDL contained less MDA and LPO than that of media that was used to culture WT-rHDL treated cells.

observed in mice treated with V156K-rHDL and R173C-rHDL than in mice treated with WT-rHDL. V156K-rHDL and R173C-rHDL were more resistant to MPO mediated oxidation in vitro and showed less cellular uptake of oxLDL by macrophages, as well as less production oxidized species in the culture media. These in vitro antioxidant abilities may contribute to the observed in vivo anti-oxidant activity, including the stimulated PON activity and FRAS ability.

PON activity is known to be important for the maintenance of the antioxidant function of $\mathrm{HDL}$ (Negre-Salvayre et al., 2006). Furthermore, $\mathrm{Ng}$ et al. (2007) recently reported that adenovirus mediated expression of human PON-3 protects against the progression of atherosclerosis in apo-E deficient mice with significant improvements of serum antioxidant capacity. In the current study, V156K-rHDL infused sera possessed the strongest PON activity (Figure $3 \mathrm{~A}$ ), which is also in agreement with the findings of our previous study regarding in vitro antioxidant activity (Cho et al., 2006), as well as the in vivo antioxidant activity observed in the current study based on the serum MPO and LPO detection and FRAS assay (Figure 4).

To determine the reason for the different inflammatory effects exerted by WT-rHDL and V156K-rHDL, we measured a susceptibility of apoA-I in rHDL to MPO treatment. In vitro oxidation mediated by leukocyte MPO, WT-rHDL was found to be more sensitive to nitration/chlorination by MPO, indica- ting that it was easily oxidized to become proinflammatory HDL (Figure 6) as explained by Fogelman (2004). The greater susceptibility of WT-rHDL to oxidation may contribute to the rapid disappearance of WT-apoA-I from mice sera, as shown in Figure 2. Moreover, V156K-rHDL or R173C-rHDL infused mice showed lower serum levels of MPO, LPO (Figure 3B), IL-6, and uric acid (Table 2) than the WT-rHDL infused mice. MPO triggers several proatherogenic effects, including the oxidation of lipoproteins (LDL and HDL) and apoA-I, which attenuates its capacity to promote cholesterol efflux and causes endothelial dysfunction. In addition, MPO can produce hypochlorous acid $(\mathrm{HOCl})$ and peroxynitrite $\left(\mathrm{ONOO}^{-}\right)$during the inflammatory process in macrophages through reactive oxygen species such as superoxide and hydrogen peroxide. $\mathrm{HOCl}$ is recognized as an important factor in atherogenesis because enzymatically active MPO and lipoproteins that have been modified by $\mathrm{HOCl}$ have been found in human atherosclerotic lesions (Daugherty et al., 1994).

In summary, blood infusions of rHDL exerted several beneficial effects in atherosclerotic apo-E deficient mice, and V156K-rHDL and R173C-rHDL were responsible for the majority of these therapeutic effects. Specifically, potent regression activity was observed in the reduction of the lipid-stained area and the immuno-detected macrophage area in the atheroma region of the aortic sinus. With the regression effect, significantly reduced GOT, GPT, IL-6, uric acid, MPO and LPO serum levels were 
detected at $48 \mathrm{~h}$ post-injection, thereby indicating the presence of an anti-inflammatory effect. Furthermore, V156K-rHDL and R173C-rHDL were detected more protein amount than that of WT-rHDL at 24 and $48 \mathrm{~h}$ post-injection. Enhanced PON activity and FRAS ability was observed in the V156K-rHDL and R173C-rHDL groups when compared with the WT-rHDL group. Finally, the V156K-rHDL and R173C-rHDL exhibited more resistance to in vitro MPO-mediated oxidation and less production of proatherogeic species in macrophage cells in the presence of oxLDL, as well as decreased cellular uptake of oxLDL into macrophages.

\section{Methods}

\section{Apo-E deficient mice and therapeutic intervention}

Male apo-E deficient mice with a C57BL/6J (5 week-old, apoe ${ }^{\text {tm1 Unc }}$ ) background were purchased from Jackson Laboratories (Bar Harbor, MA), and maintained on a normal chow diet (CRF-1, Oriental Yeast, Tokyo, Japan) that consisted of $53.5 \%$ (wt/wt) carbohydrate, $5.9 \%$ (wt/wt) fat, $23.1 \%(\mathrm{wt} / \mathrm{wt})$ protein, and $3.3 \%(\mathrm{wt} / \mathrm{wt})$ dietary fiber. After 1 week of acclimation to the chow diet, a Western diet that consisted of $0.15 \%$ cholesterol and $15 \%$ lard were added to the CRF-1 diet, which was then provided ad libitum for 15 weeks, coupled with free access to water. The mice were then randomly divided into 4 groups (5 mice/cage) and administered with $\mathrm{rHDL}$ in $0.4 \mathrm{ml}$ of tris-buffered saline (TBS) via the tail vein, as described in Table 1. All procedures were approved by the Committee of Animals for Research at Yeungnam Univeristy (Gyeongsan, South Korea).

\section{Sample collection and analysis}

After injection, a small volume of blood $(0.1 \mathrm{ml})$ was collected at $24 \mathrm{~h}$ from the retroorbital plexus via the heparinized microhematocrit capillaries (Sigma), and the mice were bled until death at $48 \mathrm{~h}$ post-injection. The serum total cholesterol (TC), HDL-cholesterol (HDL-C), triglyceride (TG), glutamic oxaloacetic transaminase (GOT), and $\gamma$-glutamic pyruvic transaminase $(\gamma$-GPT) concentrations were then determined using a Hitachi 7020 automatic blood analyzer (Hitachi Co, Tokyo, Japan), and the serum uric acid concentration was determined using the procedure described by Caraway (1955). Serum lipid hydroperoxide (LPO) was determined colorimetrically by a commercial kit (Cat\# 437639, Calbiochem, San Diego, CA) in according to manufacturer's suggestion.

\section{Fatty streak lesion analysis}

Aortic lesion formation was assessed using the method described by Paigen et al. (1987) with slight modification (Gulledge et al., 2003). Briefly, after the mice were bled to death, their hearts were perfused via the left ventricle with $30 \mathrm{ml}$ of $4 \%$ paraformaldehyde, and then fixed for at least $24 \mathrm{~h}$. The isolated upper heart/aortic sinus was then em- bedded in Tissue-Tek OCT compound (Fisher Scientific, Pittsburgh, PA) and frozen. Next, the frozen tissue blocks were positioned in a model AS620 cryotome (Shandon, Pittsburgh, PA), and $10-\mu \mathrm{m}$ serial sections of the ascending aorta were then collected on 3-aminopropyltriethoxysilane-coated slides until the aortic sinus appeared. Seven consecutive sectioned slides from each mouse were then stained with oil red-O and counterstained with hematoxylin, to visualize any fatty streak lesions, in accordance with the standard protocols. The mean area of lipid staining per section from the seven slides was then quantified using computer-assisted morphometry for each mouse using the Image Proplus software (version 4.5.1.22, Media cybernetics, Bethesda, MD).

Immunohistochemical lesion analysis was conducted using a commercially available kit (Vector Labs, Burlingame, CA) with anti-mouse macrophage antibody (MOMA-2, MCA519, Serotec, Raleigh, NC). Fluorescence-labeled secondary antibody (Cy2-conjugated-anti-lgG) was used to visualize the macrophages that were detected in the lesion area, and the samples were then observed under confocal microscopy at an excitation of $488 \mathrm{~nm}$ and an emission of 533 nm (Zeiss, LSM510 meta, Göttingen, Germany).

\section{Western blot analysis and ELISA}

In order to compare the levels of serum apolipoprotein expression, the serum pooled from each group was diluted to make equal concentrations $(5 \mathrm{mg}$ of total protein $/ \mathrm{ml})$. The equally pooled and diluted sera samples were electrophoresed via SDS-PAGE, and then subjected to Western blot analysis using the anti-proapoA-I antibody, which is capable of detecting both mouse apoA-I (28 kDa) and human proapoA-I (29 kDa). Serum interleukin (IL)-6 levels in individual mice were then measured in duplicate using an ELISA kit (MS6000B Quantikine kit, R\&D system, Minneapolis, MN). Next, the quantities of residual injected rHDLs in the mouse sera were determined using an apoA-I AUTO - N "Daiichi" kit, which contained human apoA-I polyclonal antibody (Daiichi Pure Chemicals, Tokyo, Japan), without dilution. This kit can be used to detect human proapoA-I, but not mouse apoA-I.

The serum levels of the MPO were determined using an ELISA kit that was obtained from Immunology Consultants Lab (Newberg, OR). Anti-paraoxonase antibody (Cat\# ab24261) was purchased from Abcam (Cambridge, UK). The relative band intensity $(\mathrm{BI})$ was compared via band scanning with a Gel Doc XR (Bio-Rad, Hercules, CA) using Quantity One software, version 4.5.2.

\section{Paraoxonase assay}

The paraoxonase (PON) activity toward paraoxon was measured after reaction of paraoxon hydrolysis into $p$-nitrophenol and diethylphosphate was catalyzed by the enzyme (Eckerson et al., 1983). Equally diluted serum (20 $\mu \mathrm{l}, 20 \mathrm{mg} / \mathrm{ml}$ ) was added to $230 \mu \mathrm{l}$ of the substrate (paraoxon-ethyl, Sigma Cat\# D-9286) containing solution (90 mM Tris- $\mathrm{HCl} / 3.6 \mathrm{mM} \mathrm{NaCl} / 2 \mathrm{mM} \mathrm{CaCl} 2, \mathrm{pH}$ 8.5). A PON activity of $1 \mathrm{U} / \mathrm{L}$ is defined as $1 \mathrm{mmol}$ of $p$-nitrophenol formed per minute, and the molar extinction coefficient of $p$-nitrophenol is $17,000 \mathrm{M}^{-1}, \mathrm{~cm}^{-1}$. 


\section{Ferric reducing ability of serum assay}

In order to compare the in vivo antioxidant ability of mouse serum among the groups, the ferric reducing ability of serum (FRAS) was determined as described by Benzie and Strain (1996) with slight modification (Cho et al., 2007).

\section{Purification of apolipoprotein and rHDL synthesis}

Wildtype proapoA-I and its variants were expressed and purified as previously described (Han et al., 2005). Briefly, reconstituted $\mathrm{HDL}$ (rHDL) was prepared via sodium cholate dialysis using an initial palmitoyloleoyl phosphatidylcholine (POPC):cholesterol:apoA-I:sodium cholate molar ratio of 95:5:1:150. After synthesis, the removal and quantification of endotoxins was conducted as previously described (Cho et al., 2006). Final molar composition of WT, V156K, $\mathrm{R} 173 \mathrm{C}$ was $100 \pm 8: 5: 1, \quad 103 \pm 9: 5: 1, \quad 102 \pm 10: 5: 1$ (POPC:cholesterol:apoA-I), correspondingly, from phosphorous and cholesterol determination as reported previously (Han et al., 2005).

\section{In vitro oxidation of apoA-I in the lipid-bound state}

MPO mediated apoA-I oxidation reactions were carried out following the protocol described by Shao et al. (2005) with slight modification. Briefly, for the MPO- $\mathrm{H}_{2} \mathrm{O}_{2}$-nitrite system, 1 unit of MPO from human leukocytes (minimum 50 units/mg protein, Sigma 6908), $100 \mu \mathrm{M}$ sodium nitrite, and $25 \mu \mathrm{M} \mathrm{H}_{2} \mathrm{O}_{2}$ were added to a $5 \mu \mathrm{M}$ apoA-I reaction mixture. For the MPO- $\mathrm{H}_{2} \mathrm{O}_{2}$-chloride system, 1 unit of MPO and 100 $\mathrm{mM}$ of $\mathrm{NaCl}$ were added to a $5 \mu \mathrm{M}$ apoA-I mixture. All reactions were conducted at $37^{\circ} \mathrm{C}$ in $20 \mathrm{mM}$ phosphate buffer containing $100 \mu \mathrm{M}$ diethylene triamine pentaacetic acid. The concentrations of $\mathrm{ONOO}^{-}, \mathrm{HOCl}$, and $\mathrm{H}_{2} \mathrm{O}_{2}$ were then determined spectrophotometrically $\left(\varepsilon_{302}=1670 \mathrm{M}^{-1} \mathrm{~cm}^{-1}\right.$, $\varepsilon_{292}=350 \mathrm{M}^{-1} \mathrm{~cm}^{-1}, \varepsilon_{240}=39.4 \mathrm{M}^{-1} \mathrm{~cm}^{-1}$, respectively). After oxidation, aliquots of the samples were analyzed by $12 \%$ SDS-PAGE and $8-25 \%$ native-polyacrylamide gradient gel electrophoresis (PAGGE) to compare the protein degradation patterns and the mobility of the rHDL particles, respectively.

\section{In vitro $L D L$ oxidation and cell culture}

LDL $(1.019<\mathrm{d}<1.063)$ was isolated by ultracentrifugation from human plasma using a Himac CP90 $\alpha$ (Hitachi, Tokyo, Japan) and then dialyzed against PBS. Oxidized LDL (oxLDL) was produced by incubation with $\mathrm{CuSO}_{4}$ (final 10 $\mu \mathrm{M})$ for $4 \mathrm{~h}$ at $37^{\circ} \mathrm{C}$ and then filtered $(0.2 \mu \mathrm{m})$ prior to use. A thiobarbituric acid reacting substances (TBARS) assay was then used to evaluate the oxidation of oxLDL.

THP-1 cells, a human monocytic cell line, were obtained from the American Type Culture Collection (ATCC, \#TIB-202, Manassas, VA) and maintained in RPMI1640 medium (Hyclone) supplemented with 10\% FBS until needed. Cells that had undergone no more than 20 passages were incubated in medium containing PMA (final $150 \mathrm{nM}$ ) in 24 well plates for $24 \mathrm{~h}$ at $37^{\circ} \mathrm{C}$ in a humidified incubator $\left(5 \% \mathrm{CO}_{2}, 95 \%\right.$ air) to induce differentiation into macrophages. The differentiated and adhered macrophages were then rinsed with warm PBS and incubated with $400 \mu \mathrm{l}$ of fresh RPMI1640 medium containing 1\% FBS, $50 \mu \mathrm{l}$ of oxLDL (1 $\mathrm{mg}$ of protein $/ \mathrm{ml}$ in PBS), and $50 \mu$ of PBS or each $\mathrm{rHDL}\left(1.4 \mathrm{mg}\right.$ of protein $/ \mathrm{mL}$ in PBS) for $48 \mathrm{~h}$ at $37^{\circ} \mathrm{C}$ in a humidified incubator. After incubation, the cells were washed with PBS three times and then fixed in $4 \%$ paraformaldehyde for $10 \mathrm{~min}$. Next, the fixed cells were rinsed with $100 \%$ polypropylene glycol, stained with oil-red O staining solution $(0.67 \%)$, and then washed with distilled water. THP-1 macrophage-derived foam cells were then observed and photographed using a Nikon Eclipse TE2000 microscope (Tokyo, Japan) at $600 \times$ magnification. The cell media was then analyzed by TBARS assay and a LPO assay kit (Calbiochem) to determine change of oxidized species level.

\section{Statistics}

All data were expressed as the mean \pm S.D. The data were evaluated via one-way analysis of variance (ANOVA) using an SPSS program (version 12.0, Chicago, IL), and the differences between the means were assessed using Duncan's multiple-range test. Statistical significance was defined as $P<0.05$.

\section{Supplemental data}

Supplemental Data include a figure and can be found with this article online at http://e-emm.or.kr/article/article_files/ SP-41-6-06.pdf.

\section{Acknowledgements}

This work was supported by the Korea Science and Engineering Foundation (KOSEF) grant funded by the Korea government (MEST) ((R13-2005-005-01003-0 (2008)).

\section{References}

Alam K, Meidell RS, Spady DK. Effect of up-regulating individual steps in the reverse choleterol transport pathway on reverse cholesterol transport in normolipidemic mice. $\mathrm{J}$ Biol Chem 2001;276:15641-9

Ansell BJ, Watson KE, Fogelman AM, Navab M, Fonarow GC. High density lipoprotein function. J Am Coll Cardiol 2005;46:1792-8

Barter PJ, Nicholls S, Rye KA, Anantharamaiah GM, Navab M, Fogelman AM. Antiinflammatory properties of HDL. Circ Res 2004;95:764-72

Benzie IF, Strain JJ. The ferric reducing ability of plasma (FRAP) as a measure of antioxidant power: the FRAP assay. Anal Biochem 1996;239:70-6

Caraway WT. Determination of uric acid in serum by a carbonate method. Am J Clin Pathol 1955;25:840-5

Chiesa G, Monteggia E, Marchesi M, Lonrenzon P, Laucello M, Lorusso V, Di Mario C, Karvouni E, Newton RS, Bisgaier CL, Franceschini G, Sirtori CR. Recombinant apolipoprotein A-IMilano infusion into rabbit carotid artery rapidly removes lipid from fatty streaks. Circ Res 2002;90:974-80 
Cho KH, Park SH, Han JM, Kim HC, Choi YK, Choi I. ApoA-I mutants $\mathrm{V} 156 \mathrm{~K}$ and $\mathrm{R} 173 \mathrm{C}$ promote anti-inflammatory function and antioxidant activities. Eur J Clin Invest 2006; 36:875-82

Cho KH, Park SH, Han JM, Kim HC, Chung YJ, Choi I, Kim JR. A point mutant of apolipoprotein A-I, V156K, exhibited potent anti-oxidant and anti-atherosclerotic activity in hypercholesterolemic C57BL/6 mice. Exp Mol Med 2007;39: 160-9

Coutinho TA, Turner ST, Peyser PA, Bielak LF, Sheedy PF, Kullo IJ. Associations of serum uric acid with markers of inflammation, metabolic syndrome, and subclinical coronary atherosclerosis. Am J Hypertens 2007;20:83-9

Daugherty A, Dunn JL, Rateri DL, Heinecke JW. Myeloperoxidase, a catalyst for lipoprotein oxidation, is expressed in human atherosclerotic lesions. J Clin Invest 1994;94: 437-44

Eckerson HW, Wyte CM, La Du BN. The human serum paraoxonase/arylesterase polymorphism. Am J Hum Genet 1983;35:1126-38

Fogelman AM. When good cholesterol goes bad. Nature Med 2004;10:902-3

Gordon T, Castelli WP, Hjortland MC, Kannel WB, Dawber TR. High density lipoprotein as a protective factor against coronary heart disease. Am J Med 1977;62: 707-14

Gulledge AA, Mcshea C, Schwartz T, Koch G, Lord ST. Effects of hyperfibrinogenemia on vasculature of C57BL/6 mice with and without atherogenic diet. Arterioscler Thromb Vasc Biol 2003;23:130-5

Han JM, Jeong TS, Lee WS, Choi I, Cho KH. Structural and functional properties of $\mathrm{V} 156 \mathrm{~K}$ and A158E mutants of apolipoprotein A-I in the lipid-free and lipid-bound states. J Lipid Res 2005;46:589-96

Kaul S, Rukshin V, Santos R, Azarbal B, Bisgaier CL, Johansson J, Tsang VT, Chyu KY, Cercek B, Mirocha J, Shah PK. Intramural delivery of recombinant apolipoprotein A-IMilano/Phospholipid complex (ETC-216) inhibits in-stent stenosis in porcine coronary arteries. Circulation 2003; 107:2551-4

Linsel-Nitschke P, Tall AR. HDL as a target in the treatment of atherosclerotic cardiovascular disease. Nat Rev Drug Discov 2005;4:193-205

Miyazaki A, Sakuma S, Morikawa W, Takiue T, Miake F, Terano T, Sakai M, Hakamata H, Sakamoto Y, Naito M, Ruan Y, Takahashi K, Ohta T, Horiuchi S. Intravenous injection of rabbit apolipoprotein A-I inhibits the progression of atherosclerosis in cholesterol-fed rabbits. Arterioscler Thromb Vasc Biol 1995;15:1882-8

Nanjee MN, Doran JE, Lerch PG, Miller NE. Acute effects of intravenous infusion of apoA1/phosphatidylcholine discs on plasma lipoprotein in humans. Arterioscler Thromb Vasc Biol 1999;19:979-89

Nanjee MN, Cooke CJ, Garvin R, Semeria F, Lewis G, Olszewski WL, Miller NE. Intravenous apoA-l/lecithin discs increase pre-beta-HDL concentration in tissue fluid and stimulate reverse cholesterol transport in humans. J Lipid Res 2001;42:1586-93

Navab M, Anantharamaiah GM, Reddy ST, Van Lenten BJ, Datta G, Garber D, Fogelman AM. Human apolipoprotein A-I and A-I mimetic peptides: potential for atherosclerosis reversal. Curr Opin Lipidol 2004;15:645-9

Negre-Salvayre A, Dousset N, Ferretti G, Bacchetti T, Curatola G, Salvayre T. Antioxidant and cytoprotective properties of high-density lipoproteins in vascular cells. Free Radical Biology \& Medicine 2006;41:1031-40

Newton RS, Krause BR. HDL therapy for the acute treatment of atherosclerosis. Atherosclerosis Suppl 2002;3:31-8

$\mathrm{Ng}$ CJ, Bourquard N, Hama SY, Shih D, Grijalva VR, Navab M, Fogelman AM, Reddy ST. Adenovirus-mediated expression of human paraoxonase 3 protects against the progression of atherosclerosis in apolipoprotein E-deficient mice. Arterioscler Thromb Vasc Biol 2007;27:1368-74

Nicholls SJ, Hazen SL. Myeloperoxidase and cardiovascular disease. Arterioscler Thromb Vasc Biol. 2005a;25:1102-11

Nicholls SJ, Dusting GJ, Cutri B, Bao S, Drummond GR, Rye KA, Barter PJ. Reconstituted high-density lipoproteins inhibit the acute pro-oxidant and pro-inflammatory vascular changes induced by a periarterial collar in normocholesterolemic rabbits. Circulation 2005b;111:1543-50

Nissen SE, Tsunoda T, Tuzcu EM, Schoenhagen P, Cooper CJ, Yasin M, Eaton GM, Lauer MA, Sheldon WS, Grines CL, Halpern S, Crowe T, Blankenship JC, Kerensky R. Effect of recombinant apoA-I Milano on coronary atherosclerosis in patients with acute coronary syndromes. J Am Med Assoc 2003;290:2292-300

Paigen B, Morrow A, Holmes PA, Mitchell D, Williams RA. Quantitative assessment of atherosclerotic lesions in mice. Atherosclerosis 1987;68:231-40

Ross R. Atherosclerosis-an inflammatory disease. N Eng J Med 340:115-26

Shah PK, Nilsson J, Kaul S, Fishbein MC, Ageland H, Hamsten A, Johansson J, Karpe F, Cercek B. Effects of recombinant apolipoprotein A-IMilano on aortic atherosclerosis in apo-E deficient mice. Circulation 1998;97:780-5

Shah PK, Yano J, Reyes O, Chyu KY, Kaul S, Bisgaier CL, Drake S, Cercek B. High-dose recombinant apolipoprotein A-IMilano mobilizes tissue cholesterol and rapidly reduces plaque lipid and macrophage content in apolipoprotein E-deficient mice. Circulation 2001;103:3047-50

Shao B, Bergt C, Fu X, Green P, Voss JC, Oda MN, Oram JF, Heinecke JW. Tyr192 in apolipoprotein A-I is the major site of nitration and chlorination by myeloperoxidase, but only chlorination markedly impairs ABCA1-dependent cholesterol transport. J Biol Chem 2005;280:5983-93

Shao B, Oda MN, Bergt C, Fu X, Green P, Brot N, Oram JF, Heinecke JW. Myeloperoxidase impairs ABCA1-dependent cholesterol efflux through methionine oxidation and site-specific tyrosine chlorination of apolipoproteinA-I. J Biol 


\section{Chem 2006;281:9001-4}

Tardif JC, Gregoire J, L'Allier PL, Ibrahim R, Lesperance J, Heinonen TM, Kouz S, Berry C, Basser R, Lavoie MA,
Guertin MC, Rodes-Cabau J. Effects of reconstituted high-density lipoprotein infusions on coronary atherosclerosis. J Am Med Assoc 2007:297:1675-82 